\title{
IWSECO 2011: Third International Workshop on Software Ecosystems
}

\author{
Slinger Jansen ${ }^{1}$, Jan Bosch ${ }^{2}$, Faheem Ahmed ${ }^{3}$, and Piers Campbell ${ }^{3}$ \\ ${ }^{1}$ Utrecht University, The Netherlands \\ ${ }^{2}$ Intuit Inc, USA \\ ${ }^{3}$ United Arab Emirates University
}

Software vendors no longer function as independent units, where all customers are end-users, where there are no suppliers, and where all software is built in-house. Instead, software vendors have become networked, i.e., software vendors are depending on (communities of) service and software component suppliers, value-added-resellers, and pro-active customers who build and share customizations. Software vendors now have to consider their strategic role in the software ecosystem to survive. With their role in the software ecosystem in mind, software vendors can become more successful by opening up their business, devising new business models, forging long-lasting relationships with partnership networks, and overcoming technical and social challenges that are part of these innovations. The focus of the first workshop was the definition of the research field. The second workshop's focus was the 'ideal' architecture of a software platform. The third workshop on software ecosystems focuses on the management of software ecosystems, i.e., how a software vendor can manage its network of partners, developers, service deliverers, and other third parties that play a role in the software ecosystem.

Typically, software vendors have several instruments available to them for managing their ecosystem, such as the creation of partnership models or the introduction of component and service certification. The effects of these decisions on the software ecosystem have not yet been made measurable, which can be considered one of the main challenges of the field of software ecosystems.

A software ecosystem is a set of actors functioning as a unit and interacting with a shared market for software and services, together with the relationships among them. These relationships are frequently underpinned by a common technological platform or market and operate through the exchange of information, resources and artifacts. Several challenges lie in the research area of software ecosystems. To begin with, insightful and scalable modeling techniques for software ecosystems currently do not exist. Furthermore, methods are required that enable software vendors to transform their legacy architectures to accommodate reusability of internal common artifacts and external components and services. Finally, methods are required that support software vendors in choosing survival strategies in software ecosystems.

The workshop aims to further increase the body of knowledge in this specific area of software reuse and software engineering by providing a forum to exchange ideas and discuss state-of-the-art results. It will build and shape the community of leading practitioners and research experts. Given the relevance of software ecosystems, and the rather unexplored scientific and industry contribution in this field, the workshop will deliver a state-of-the-practice overview of the available knowledge on software ecosystems, as well as an overview of challenges for further research. 